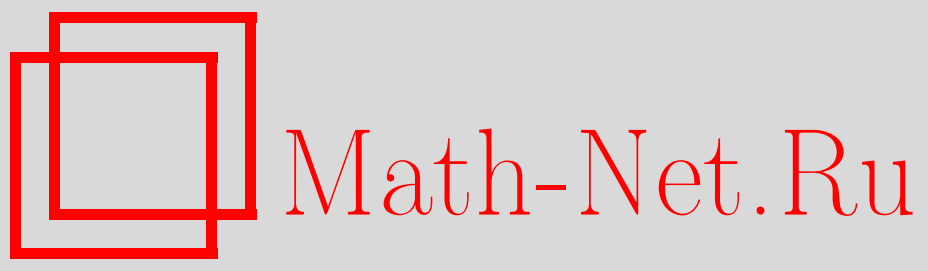

С. М. Гусейн-Заде, Ф. Дельгадо, А. Кампильо, Универсальные абелевы накрытия рациональных особенностей поверхностей и мультииндексные фильтрации, Функи.. анализ и его прил., 2008, том 42, выпуск 2, 3-10

DOI: https://doi.org/10.4213/faa2897

Использование Общероссийского математического портала MathNet.Ru подразумевает, что вы прочитали и согласны с пользовательским соглашением

http: //www . mathnet.ru/rus/agreement

Параметры загрузки:

IP : 18.207 .199 .55

26 апреля 2023 г., $07: 12: 30$

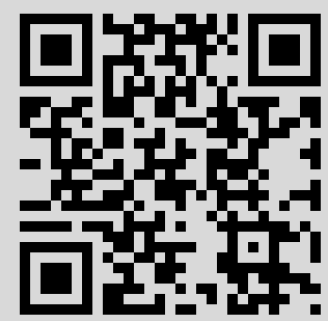


Функционалъный анализ и его приложения

2008, т. 42, вып. 2, с. 3-10

УДК 512.774.1

\title{
Универсальные абелевы накрытия рациональных особенностей поверхностей и мультииндексные фильтрации*
}

\author{
(c) 2008. С. М. ГУСЕЙН-ЗАДЕ, Ф. ДЕЛЬГАДО, А. КАМПИЛЬО
}

В [2] и [3] были вычислены ряды Пуанкаре некоторых (мультииндексных) фильтраций на кольце ростков функций на рациональной особенности поверхности. Эти ряды Пуанкаре были выражены в виде целых частей некоторых дробностепенных рядов, интерпретация которых отсутствовала. В настоящей работе показывается, что с точностью до простой замены переменных эти дробностепенные ряды являются «редукцией» эквивариантных рядов Пуанкаре для некоторых фильтраций на кольце $\mathcal{O}_{\widetilde{\delta}, 0}$ ростков функций на универсальном абелевом накрытии $(\widetilde{\mathcal{S}}, 0)$ поверхности $(\mathcal{S}, 0)$. Мы вычисляем эти эквивариантные ряды Пуанкаре. С другой точки зрения универсальные абелевы накрытия рациональных особенностей поверхностей изучались в [7].

Пусть $(\mathcal{S}, 0)$ - изолированная рациональная особенность комплексной поверхности, и пусть $\pi:(X, \mathcal{D}) \rightarrow(\mathcal{S}, 0)$ - ее разрешение (не обязательно минимальное). Здесь $X$ - неособая комплексная поверхность, $\pi$ - собственное аналитическое отображение, являющееся изоморфизмом вне начала координат в $\mathcal{S}$, исключительный дивизор $\mathcal{D}=\pi^{-1}(0)$ является дивизором с нормальными пересечениями на поверхности $X$, все компоненты $E_{\sigma}(\sigma \in \Gamma)$ исключительного дивизора $\mathcal{D}$ изоморфны комплексной проективной прямой $\mathbb{C P}^{1}$ и (двойственный) граф разрешения является деревом.

Пусть $\mathcal{O}_{\mathcal{S}, 0}$ - кольцо ростков аналитических функций на поверхности $(\mathcal{S}, 0)$. Для $\sigma \in \Gamma$, т.е. для компоненты $E_{\sigma}$ исключительного дивизора $\mathcal{D}$, и для $f \in \mathcal{O}_{\mathcal{S}, 0}$ обозначим через $v_{\sigma}(f)$ порядок нуля поднятия $f \circ \pi$ функции $f$ на пространство $X$ разрешения вдоль компоненты $E_{\sigma}$. Выберем несколько компонент $E_{1}, \ldots, E_{s}$ исключительного дивизора $\mathcal{D}(\{1, \ldots, s\} \subset \Gamma)$. Нормирования $v_{1}, \ldots, v_{s}$ определяют мультииндексную фильтрацию $\{J(\underline{v})\}$ на кольце $\mathcal{O}_{s, 0}:$ для $\underline{v}=\left(v_{1}, \ldots, v_{s}\right) \in \mathbb{Z}^{s}$ имеем $J(\underline{v}):=\left\{f \in \mathcal{O}_{s, 0}: \underline{v}(f) \geqslant \underline{v}\right\}$ (здесь $\underline{v}(f)=\left(v_{1}(f), \ldots, v_{s}(f)\right) \in \mathbb{Z}^{s}$ и $\underline{v}^{\prime} \geqslant \underline{v}$, если $v_{i}^{\prime} \geqslant v_{i}$ для всех $\left.i=1, \ldots, s\right)$. (Достаточно определить $J(\underline{v})$ только для неотрицательных $\underline{v}$, т. е. для $\underline{v} \in \mathbb{Z}_{\geqslant 0}^{s}$. Однако для приведенного ниже определения удобно считать, что $\underline{v} \in \mathbb{Z}^{s}$.)

Определение ряда Пуанкаре мультииндексной фильтрации можно найти, например, в [2]-[4]. Для одноиндексной фильтрации ряд Пуанкаре - это произво-

* Исследование частично поддержано грантами РФФИ-007-00593, INTAS-05-7805, NWORFBR 047.011.2004.026 и RFBR-JSPS 06-01-91063 (С. М. Гусейн-Заде) и грантом МТМ200764704 (Ф. Дельгадо, А. Кампильо). 
дящий ряд для размерностей факторов в фильтрации:

$$
P(t)=\sum_{v=0}^{\infty} \operatorname{dim}(J(v) / J(v+1)) t^{v} .
$$

Для мультииндексной фильтрации $\{J(\underline{v})\}$ надо взять факторы $J(\underline{v}) / J\left(\underline{v}+\underline{1}_{i}\right)$, где $\underline{1}_{i}=(0, \ldots, 0,1,0, \ldots, 0)$ (1 находится на $i$-м месте), и просуммировать их размерности с использованием формулы включения-исключения. Это приводит к следующему определению. Пусть $L\left(t_{1}, \ldots, t_{s}\right)=\sum_{\underline{v} \in \mathbb{Z}^{s}} \operatorname{dim}(J(\underline{v}) / J(\underline{v}+\underline{1})) \cdot \underline{t}^{\underline{v}}$, $\underline{1}=(1, \ldots, 1)$, ряд Лорана от переменных $t_{1}, \ldots, t_{s}$ (вообще говоря, бесконечный во всех направлениях). Легко понять, что вдоль любой прямой решетки $\mathbb{Z}^{s}$, параллельной координатной оси, коэффициент при мономе $\underline{t} \underline{v}$ один и тот же для всех $\underline{v}$ из неположительной части прямой. Отсюда следует, что

$$
P^{\prime}\left(t_{1}, \ldots, t_{s}\right)=L\left(t_{1}, \ldots, t_{s}\right) \cdot \prod_{i=1}^{s}\left(t_{i}-1\right)
$$

является степенным рядом от $t_{1}, \ldots, t_{s}$, т. е. элементом кольца $\mathbb{Z}\left[\left[t_{1}, \ldots, t_{s}\right]\right]$. Степенной ряд

$$
P_{\mathcal{S}}\left(t_{1}, \ldots, t_{s}\right)=\frac{P^{\prime}\left(t_{1}, \ldots, t_{s}\right)}{t_{1} \cdots t_{s}-1}
$$

называется рядом Пуанкаре фильтрации $\{J(\underline{v})\}$.

Это определение может быть переформулировано в терминах интеграла по эйлеровой характеристике следующим образом. Пусть $\mathbb{P} \mathcal{O}_{\mathcal{S}, 0}$ - проективизация кольца (векторного пространства) $\mathcal{O}_{\mathcal{S}, 0}$. Интегрирование по отношению к эйлеровой характеристике по пространству $\mathbb{P} \mathcal{O}_{\mathcal{S}, 0}$ было определено, например, в [1], [2]. Будем рассматривать $\underline{t} \underline{v}$ как функцию на пространстве $\mathbb{P} \mathcal{O}_{\mathcal{S}, 0}$ со значениями в группе $\mathbb{Z}\left[\left[t_{1}, \ldots, t_{s}\right]\right]$ степенных рядов от $t_{1}, \ldots, t_{s}$ (со сложением в качестве групповой операции). Можно показать, что

$$
P_{\mathcal{S}}\left(t_{1}, \ldots, t_{s}\right)=\int_{\mathbb{P} \mathcal{O}_{\mathcal{S}, 0}} \underline{t}^{\underline{v}} d \chi
$$

(это следует из того факта, что для конечномерного комплексного векторного пространства эйлерова характеристика его проективизации равна его размерности).

В [2] был вычислен ряд Пуанкаре $P\left(t_{1}, \ldots, t_{s}\right)$ описанной (дивизориальной) фильтрации на кольце $\mathcal{O}_{\mathcal{S}, 0}$. Пусть $\left(E_{\sigma} \circ E_{\delta}\right)$ - матрица пересечений компонент исключительного дивизора. Для $\sigma \neq \delta$ индекс пересечения $E_{\sigma} \circ E_{\delta}$ равен 1 , если компоненты $E_{\sigma}$ и $E_{\delta}$ пересекаются (в точке), и нулю, если они не пересекаются; индекс самопересечения $E_{\sigma} \circ E_{\sigma}$ каждой компоненты $E_{\sigma}$ - отрицательное целое число. Пусть $d=\operatorname{det}\left(-\left(E_{\sigma} \circ E_{\delta}\right)\right)$, и пусть $\left(m_{\sigma \delta}\right)=-\left(E_{\sigma} \circ E_{\delta}\right)^{-1}$. Все элементы $m_{\sigma \delta}$ положительны, и $m_{\sigma \delta} \in(1 / d) \mathbb{Z}$. Для $\sigma \in \Gamma$ положим $\underline{m} \sigma:=$ $\left(m_{\sigma 1}, \ldots, m_{\sigma s}\right) \in \mathbb{Q}_{\geqslant 0}^{s}$. Пусть $\dot{E}_{\sigma}-\ll$ «еособая часть» компоненты $E_{\sigma}$ в исключительном дивизоре $\mathcal{D}$, т. е. $E_{\sigma}$ без точек пересечения со всеми остальными компонентами исключительного дивизора $\mathcal{D}$. 
Для дробностепенного ряда $S\left(t_{1}, \ldots, t_{s}\right) \in \mathbb{Z}\left[\left[t_{1}^{1 / d}, \ldots, t_{s}^{1 / d}\right]\right]$ обозначим через Int $S\left(t_{1}, \ldots, t_{s}\right)$ его «целую часть», т. е. сумму всех его мономов с целыми показателями степеней. В [2] было показано, что

$$
P\left(t_{1}, \ldots, t_{s}\right)=\operatorname{Int} \prod_{\sigma \in \Gamma}\left(1-\underline{t}^{\underline{m}} \sigma\right)^{-\chi\left(\dot{E}_{\sigma}\right)},
$$

где $\underline{t} \underline{m}:=t_{1}^{m_{1}} \cdot \ldots \cdot t_{s}^{m_{s}}$, а $\chi(X)$ - эйлерова характеристика пространства $X$.

Аналогичная формула для ряда Пуанкаре мультииндексной фильтрации на кольце $\mathcal{O}_{\mathcal{S}, 0}$, определенной порядками ростка функции на неприводимых компонентах кривой $(C, 0) \subset(S, 0)$, была получена в [3].

В [2] дробностепенной ряд

$$
Q(\underline{t})=\prod_{\sigma \in \Gamma}\left(1-\underline{t}^{\underline{m}} \sigma\right)^{-\chi\left(\dot{E}_{\sigma}\right)}
$$

(или аналогичный ряд в [3]) участвовал как формальное выражение, удобное для записи формулы (1) для ряда Пуанкаре $P\left(t_{1}, \ldots, t_{s}\right)$. Интерпретация этого ряда отсутствовала.

В [4] был определен эквивариантный ряд Пуанкаре «эквивариантной» фильтрации на кольце $\mathcal{O}_{V, 0}$ ростков функций на ростке $(V, 0)$ комплексно-аналитического пространства с действием конечной группы $G$. Этот ряд Пуанкаре был вычислен для дивизориальной фильтрации на кольце $\mathcal{O}_{\mathbb{C}^{2}, 0}$ и для фильтрации, определенной ветвями $G$-инвариантной особенности плоской кривой $(C, 0) \subset$ $\left(\mathbb{C}^{2}, 0\right)$, если на плоскости $\mathbb{C}^{2}$ имеется действие (представление) группы $G$.

Пусть $p:(\widetilde{\mathcal{S}}, 0) \rightarrow(\mathcal{S}, 0)$ - универсальное абелево накрытие особенности поверхности $(\mathcal{S}, 0)$, см., например, [6], [7]. Оно может быть описано следующим образом. Пусть $G=H_{1}(\mathcal{S} \backslash\{0\} ; \mathbb{Z})$ - первая группа гомологий (неособой) поверхности $\mathcal{S} \backslash\{0\}$. Порядок группы $G$ равен определителю $d$ минус матрицы пересечений $-\left(E_{\sigma} \circ E_{\delta}\right)$, и при этом группа $G$ изоморфна коядру $\mathbb{Z}^{\Gamma} / \operatorname{Im} I$ отображения $I: \mathbb{Z}^{\Gamma} \rightarrow \mathbb{Z}^{\Gamma}$, задаваемого этой матрицей. Группа $G$ действует на ростке $(\widetilde{\mathcal{S}}, 0)$, и ограничение $\left.p\right|_{\widetilde{\mathcal{S}} \backslash\{0\}}$ отображения $p$ на дополнение к началу координат является (обычным, неразветвленным) накрытием $\widetilde{\mathcal{S}} \backslash\{0\} \rightarrow \mathcal{S} \backslash\{0\}$ со структурной группой $G$. Отображение $p$ может быть поднято до (разветвленного) накрытия $p:(\widetilde{X}, \widetilde{\mathcal{D}}) \rightarrow(X, \mathcal{D})$, где $\widetilde{X}-$ нормальная поверхность (вообще говоря, не неособая), $\widetilde{X} \backslash \widetilde{\mathcal{D}} \cong \widetilde{\mathcal{S}} \backslash\{0\}$ :

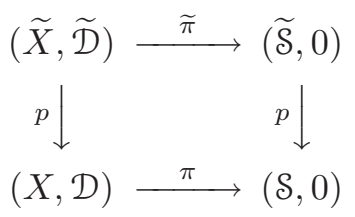

(можно определить $\widetilde{X}$ как нормализацию расслоенного произведения $X \times s \widetilde{\mathcal{S}}$ пространств $X$ и $\widetilde{\mathcal{S}}$ над $\mathcal{S})$.

Пусть $g_{\sigma}, \sigma \in \Gamma$, - элемент группы $G=H_{1}(X \backslash \mathcal{D} ; \mathbb{Z})$, представленный петлей в многообразии $X \backslash \mathcal{D}$, обходящей вокруг компоненты $E_{\sigma}$ в положительном направлении. Группа $G$ порождена элементами $g_{\sigma}$ для всех $\sigma \in \Gamma$. Для точки $x \in \dot{E}_{\sigma}$ и для точки $\widetilde{x}$ из ее прообраза $p^{-1}(x)$ локально (в окрестности точки $\widetilde{x}$ ) 
отображение $p: \widetilde{\mathcal{D}} \rightarrow \mathcal{D}$ является изоморфизмом, а отображение $p: \widetilde{X} \rightarrow X-$ разветвленным (вдоль $\mathcal{D})$ накрытием, порядок $d_{\sigma}$ которого совпадает с порядком образующей $g_{\sigma}$ группы $G$.

Лемма 1. Порядок $d_{\sigma}$ элемента $g_{\sigma} \in G$ равен наименъшему натуральному $k$, для которого $k m_{\delta \sigma}-$ челое число для всех $\delta \in \Gamma$.

Доказательство. Это следует непосредственно из того факта, что $\mathbb{Z}^{\Gamma} / \operatorname{Im} I \cong$ $\operatorname{Im} m / \mathbb{Z}^{\Gamma}$, где $m: \mathbb{Z}^{\Gamma} \rightarrow \mathbb{Q}^{\Gamma}$ - отображение, определяемое матрицей $\left(m_{\sigma \delta}\right)$ (т. е. минус обратное к отображению $I)$.

Пусть $R(G)$ - кольцо (виртуальных) представлений группы $G$. Для $\sigma \in \Gamma$ пусть $\alpha_{\sigma}$ - одномерное представление $G \rightarrow \mathbb{C}^{*}=\mathbf{G L}(1, \mathbb{C})$ группы $G$, определенное тем, что $\alpha_{\sigma}\left(g_{\delta}\right)=\exp \left(-2 \pi \sqrt{-1} m_{\sigma \delta}\right.$ ) (здесь знак минус отражает тот факт, что действие элемента $g \in G$ на кольце $\mathcal{O}_{\widetilde{\S}, 0}$ определяется формулой $\left.(g \cdot f)(x)=f\left(g^{-1}(x)\right)\right)$.

Выберем какую-либо компоненту $\widetilde{E}_{i}$ прообраза $p^{-1}\left(E_{i}\right)$ компоненты $E_{i}$, и пусть $\widetilde{v}_{i}-$ соответствующее дивизориальное нормирование на кольце $\mathcal{O}_{\widetilde{\delta}, 0}$ ростков функций на $(\widetilde{\mathcal{S}}, 0)$. На пространстве $\bigcup_{\alpha} \mathcal{O}_{\widetilde{\delta}, 0}^{\alpha}$ всех $G$-эквивариантных функций на $(\widetilde{\mathcal{S}}, 0)$ ( $\alpha$ пробегает все неэквивалентные одномерные представления группы $G$ ) нормирование $\widetilde{v}_{i}$ не зависит от выбора компоненты $\widetilde{E}_{i}$. В [4] был определен эквивариантный ряд Пуанкаре мультииндексной фильтрации, определенной дивизориальными нормированиями $\widetilde{v}_{1}, \ldots, \widetilde{v}_{s}$.

Теорема 1. Эквивариантный ряд Пуанкаре $P^{G}\left(t_{1}, \ldots, t_{s}\right)$ s-индексной филътрачии, определенной набором дивизориальных нормирований $\left\{\widetilde{v}_{1}, \ldots, \widetilde{v}_{s}\right\}, \partial а$ ется формулой

$$
\begin{aligned}
P^{G}\left(t_{1}, \ldots, t_{s}\right) & =\prod_{\sigma \in \Gamma}\left(1-\alpha_{\sigma} \underline{\underline{t}} \underline{\underline{m}}_{\sigma}\right)^{-\chi\left(\dot{E}_{\sigma}\right)} \\
& =\prod_{\sigma \in \Gamma}\left(1-\alpha_{\sigma} t_{1}^{d_{1} m_{1 \sigma}} \cdots t_{s}^{d_{s} m_{s \sigma}}\right)^{-\chi\left(\dot{E}_{\sigma}\right)} .
\end{aligned}
$$

Замечание. Эквивалентная формула для одного нормирования (т.е. для $s=1$ ), определенного дивизором, являющимся точкой разветвления графа разрешения, получена в [8] для более широкого класса особенностей поверхностей - так называемых фактор-склеенных (splice-quotient), особенностей.

Редукцией $\operatorname{red} S\left(t_{1}, \ldots, t_{s}\right)$ степенного ряда $S\left(t_{1}, \ldots, t_{s}\right)=\sum_{\underline{v} \in \mathbb{Z}_{\geqslant 0}^{s}} s_{\underline{v}} \underline{t} \underline{v} \in$ $R(G)\left[\left[t_{1}, \ldots, t_{s}\right]\right] \quad(R(G)$ - кольцо представлений группы $G)$ назовем ряд $\sum_{\underline{v} \in \mathbb{Z}_{\geqslant 0}^{s}}\left(\operatorname{dim} s_{\underline{v}}\right) \underline{t} \underline{v} \in \mathbb{Z}\left[\left[t_{1}, \ldots, t_{s}\right]\right]$.

Следствие. Имеет место равенство $\operatorname{red} P^{G}\left(t_{1}, \ldots, t_{s}\right)=Q\left(t_{1}^{d_{1}}, \ldots, t_{s}^{d_{s}}\right)$, где $Q(\underline{t})$ - дробностепенной ряд, определенный формулой (2).

Доказательство теоремы 1. Для краткости будем говорить, что эффективный дивизор на $\dot{\mathcal{D}}=\bigcup \dot{E}_{\sigma}$ (или на $\left.\dot{\widetilde{\mathcal{D}}}=p^{-1}(\dot{\mathcal{D}})\right)$ является дивизором Картье, если он есть пересечение собственного прообраза дивизора Картье на $(\mathcal{S}, 0)$ (или на $(\widetilde{\mathcal{S}}, 0))$ с $\dot{\mathcal{D}}$ (или с $\dot{\widetilde{\mathcal{D}}})$. Эквивариантный ряд Пуанкаре $P^{G}\left(t_{1}, \ldots, t_{s}\right)$ 
может быть представлен в виде интеграла по отношению к эйлеровой характеристике монома $\alpha \underline{t} \underline{\underline{v}}$ по несвязному объединению $\coprod_{\{\alpha\}} \mathbb{P} \mathcal{O}_{\widetilde{\mathcal{S}}, 0}^{\alpha}$ проективизаций пространств $\mathcal{O}_{\widetilde{\mathcal{S}}, 0}^{\alpha}$ ростков функций на $(\widetilde{\mathcal{S}}, 0)$, эквивариантных по отношению к различным одномерным представлениям $\alpha$ группы $G$. Так же, как, например, в [4], определяется отображение из $\coprod_{\{\alpha\}} \mathbb{P} \mathcal{O}_{\widetilde{\mathcal{S}}, 0}^{\alpha}$ в пространство $G$-инвариантных эффективных дивизоров на $\dot{\mathcal{D}}:$ эквивариантному ростку функции на $(\widetilde{\mathcal{S}}, 0)$ ставится в соответствие пересечение собственного прообраза дивизора его нулей с исключительным дивизором $\widetilde{\mathcal{D}}$. Из аналога предложения 2 из [2] следует, что прообраз любой точки при этом отображении является комплексным аффинным пространством и поэтому имеет эйлерову характеристику, равную единице. Отсюда вытекает, что эквивариантный ряд Пуанкаре $P^{G}(\underline{t})$ равен интегралу по эйлеровой характеристике монома $\alpha \underline{t} \underline{\widetilde{v}}$ по пространству $G$-инвариантных эффективных дивизоров Картье на $\dot{\mathcal{D}}$. Здесь $\alpha$ и $\underline{\widetilde{v}}-$ функции (в действительности гомоморфизмы полугрупп) на пространстве $G$-инвариантных дивизоров Картье на $\dot{\mathcal{D}}$ со значениями в кольце представлений $R(G)$ и решетке $\mathbb{Z}_{\geqslant 0}^{s}$ : $G$-инвариантный дивизор Картье определяет порядки нулей соответствующей ( $G$-эквивариантной) функции вдоль компонент $\dot{E}_{i}$, а также и соответствующее одномерное представление группы $G$.

Таким образом, для того чтобы вычислить эквивариантный ряд Пуанкаре $P^{G}(\underline{t})$, надо описать пространство $G$-инвариантных эффективных дивизоров Картье на $\dot{\widetilde{D}}$ и соответствующие функции $\underline{v}$ и $\alpha$ на нем.

Лемма 2. Любой G-инвариантный эффективный дивизор на $\dot{\widetilde{D}}$ является дивизором Kартве.

Доказательство. Достаточно показать это для дивизора $\sum_{\widetilde{x} \in p^{-1}(x)} \widetilde{x}$ для точки $x \in \dot{E}_{\sigma}$, т. е. для $G$-орбиты точки из $\dot{\widetilde{E}}_{\sigma}$. Группа изотропии $G_{\widetilde{x}}$ точки $\widetilde{x} \in$ $p^{-1}(x)$ является циклической подгруппой группы $G$ порядка $d_{\sigma}$, порожденной элементом $g_{\sigma}$ (этот элемент действует тривиально на $p^{-1}\left(E_{\sigma}\right)$ ).

Возьмем на $(X, \mathcal{D})$ росток неособой кривой $L_{\sigma}$, трансверсальный к исключительному дивизору $\mathcal{D}$ в точке $x$. По критерию Артина (см., например, $[9$, лемма на с. 156]) дивизор $d \cdot L_{\sigma}$ является собственным прообразом дивизора Картье на $(\mathcal{S}, 0)$ (в действительности таковым является уже $\left.d_{\sigma} L_{\sigma}\right)$, т. е. существует функция $f_{\sigma}:(\mathcal{S}, 0) \rightarrow(\mathbb{C}, 0)$, такая, что собственным прообразом дивизора $\left(f_{\sigma}=0\right)$ является $d \cdot L_{\sigma}$. Пусть $\bar{f}_{\sigma}=f_{\sigma} \circ \pi$ - поднятие функции $f_{\sigma}$ на пространство $X$ разрешения, и пусть $\widetilde{f}_{\sigma}=f_{\sigma} \circ \pi \circ p-$ поднятие функции $f_{\sigma}$ на пространство $\widetilde{X}$ модификации универсального абелева накрытия $(\widetilde{\mathcal{S}}, 0)\left(\widetilde{f}_{\sigma}\right.$ является $G$-инвариантной функцией на $\widetilde{X})$. Опишем дивизор $\left(\tilde{f}_{\sigma}=0\right)$. Пусть $\widetilde{L}_{\sigma, \widetilde{x}} \subset \widetilde{X}-$ росток в точке $\widetilde{x} \in p^{-1}(x)$ прообраза кривой $L_{\sigma} \subset X$ (относительно отображения $p$ ). 
Порядок нуля функции $\widetilde{f}_{\sigma}$ вдоль $\widetilde{L}_{\sigma, \widetilde{x}}$ равен $d$. Порядок нуля функции $\bar{f}_{\sigma}$ вдоль компоненты $\dot{E}_{\delta}$ равен $d \cdot m_{\sigma \delta}$. Порядок ветвления отображения $p$ над компонентой $\dot{E}_{\delta}$ равен $d_{\delta}$. Поэтому порядок нуля функции $\widetilde{f}_{\sigma}=\bar{f}_{\sigma} \circ p$ вдоль прообраза компоненты $\dot{E}_{\delta}$ равен $d \cdot d_{\delta} \cdot m_{\sigma \delta}$. Это (целое) число делится на $d$ (поскольку $d_{\delta} m_{\sigma \delta}$ - целое число, см. лемму 1$)$. Поэтому дивизор нулей функции $\widetilde{f}_{\sigma}$ делится на $d$, т. е. порядок нуля этой функции вдоль любой компоненты ее множества нулей делится на $d$. Это означает, что корень $\sqrt[d]{\widetilde{f}_{\sigma}}$ степени $d$ из функции $\widetilde{f}_{\sigma}$ (точнее, (любая) ветвь этого корня) есть корректно определенная с точностью до умножения на корень степени $d$ из единицы $G$-эквивариантная комплексноаналитическая функция на $\widetilde{X}$ и, таким образом, является результатом поднятия $G$-эквивариантной функции на $(\widetilde{\mathcal{S}}, 0)$ (см., например, $[5$, с. 39]).

Следствие. Любой $G$-инвариантный дивизор на универсалъном абелевом накрытии $(\widetilde{\mathcal{S}}, 0)$ рациональной особенности поверхности $(\mathcal{S}, 0)$ является дивизором Картве.

Лемма 2 означает, что пространство эффективных $G$-инвариантных дивизоров Картье на $\dot{\widetilde{\mathcal{D}}}$ изоморфно пространству всех эффективных дивизоров на $\dot{\mathcal{D}}$. Из доказательства леммы 2 следует, что порядок нуля $G$-эквивариантной функции $\widetilde{f}_{\sigma}$ (соответствующей одной точке $x \in \dot{E}_{\sigma}$ ) вдоль компоненты $\widetilde{E}_{i}$ равен $d_{i} m_{\sigma i}$. Надо найти (одномерное) представление $\alpha_{\sigma}$ группы $G$, по отношению к которому функция $\widetilde{f}_{\sigma}$ является $G$-эквивариантной.

Лемма 3. $\alpha_{\sigma}\left(g_{\delta}\right)=\exp \left(-2 \pi \sqrt{-1} m_{\sigma \delta}\right)$.

Доказательство. Элемент $g_{\delta}$ группы $G$ действует тривиально на прообразе $p^{-1}\left(\dot{E}_{\delta}\right)$ компоненты $\dot{E}_{\delta}$ исключительного дивизора и действует умножением на $\exp \left(\frac{2 \pi}{d_{\delta}} \sqrt{-1}\right)$ на нормальном направлении к ней. Порядок нуля функции $\widetilde{f}_{\sigma}$ вдоль прообраза $p^{-1}\left(\dot{E}_{\delta}\right)$ равен $m_{\sigma \delta} d_{\delta}$. Поэтому

$$
\frac{g_{\delta} \cdot f_{\sigma}}{f_{\sigma}}=\exp \left(-\frac{2 \pi \sqrt{-1} m_{\sigma \delta} d_{\delta}}{d_{\delta}}\right)=\exp \left(-2 \pi \sqrt{-1} m_{\sigma \delta}\right) .
$$

Теперь теорема 1 следует из обычных рассуждений, используемых, например, в [2], [4]. Пространство эффективных дивизоров на $\dot{\mathcal{D}}=\bigcup \dot{E}_{\sigma}$ является прямым произведением пространств эффективных дивизоров на компонентах $\dot{E}_{\sigma}$. Каждое из этих пространств является несвязным объединением симметрических степеней $S^{k} \dot{E}_{\sigma}$ компонент $\dot{E}_{\sigma}$. Поэтому

$$
P^{G}\left(t_{1}, \ldots, t_{s}\right)=\prod_{\sigma \in \Gamma}\left(\sum_{k=0}^{\infty} \chi\left(S^{k} \dot{E}_{\sigma}\right) \cdot \alpha_{\sigma}^{k} \underline{t}^{k \underline{d} \underline{m}_{\sigma}}\right)
$$

(это следует из того, что $\underline{v}$ и $\alpha$ являются гомоморфизмами полугрупп). Теперь (3) вытекает из известной формулы

$$
\sum_{k=0}^{\infty} \chi\left(S^{k} X\right) \cdot t^{k}=(1-t)^{-\chi(X)} .
$$


Аналогичный результат имеет место для фильтрации на кольце $\mathcal{O}_{\widetilde{\mathcal{S}}, 0}$, определенной порядками ростка функции на ветвях $G$-инвариантной кривой $(\widetilde{C}, 0) \subset$ $(\widetilde{\mathcal{S}}, 0)$. Пусть $\widetilde{C}=\bigcup_{i=1}^{r} \widetilde{C}_{i}$, где $\widetilde{C}_{i}$ суть $G$-неприводимые $G$-инвариантные компоненты кривой $\widetilde{C}$ (вообще говоря, каждая кривая $\widetilde{C}_{i}$ состоит из нескольких неприводимых компонент, переставляемых элементами группы $G)$. Kaждая кривая $\widetilde{C}_{i}$ является прообразом относительно отображения $p$ неприводимой кривой $C_{i}$ на поверхности $(\mathcal{S}, 0)$. Кривая $\widetilde{C}=\bigcup_{i=1}^{r} \widetilde{C}_{i}$ определяет $r$-индексную фильтрацию на пространстве $\bigcup_{\alpha} \mathcal{O}_{\widetilde{\mathcal{S}}, 0}^{\alpha} \quad G$-эквивариантных функций на поверхности $(\widetilde{\mathcal{S}}, 0)$ (или на пространстве $\bigcup_{\alpha} \mathcal{O}_{\widetilde{C}, 0}^{\alpha} \quad G$-эквивариантных функций на кривой $(\widetilde{C}, 0))$. Пусть $\varphi_{i}:(\mathbb{C}, 0) \rightarrow(\widetilde{\mathcal{S}}, 0)-$ параметризация (униформизация) неприводимой компоненты кривой $\widetilde{C}_{i}$. Для ростка функции $f$ на $(\widetilde{\mathcal{S}}, 0)$ обозначим через $\widetilde{w}_{i}(f)$ порядок нуля функции $f \circ \varphi_{i}$ в начале координат: $f \circ \varphi_{i}(\tau)=$ $a \tau^{\widetilde{w}_{i}(f)}+$ члены более высокой степени, $a \neq 0$. Нормирования $\widetilde{w}_{1}, \ldots, \widetilde{w}_{r}$ обычным образом определяют мультииндексную фильтрацию на кольце функций $\operatorname{Ha}(\widetilde{\mathcal{S}}, 0)$.

Пусть $\pi:(X, \mathcal{D}) \rightarrow(\mathcal{S}, 0)$ - разрешение особенности поверхности $(\mathcal{S}, 0)$, которое в то же время является разрешением («embedded resolution») кривой $(C, 0) \subset(\mathcal{S}, 0), C=\bigcup_{i=1}^{r} C_{i}$. Пусть $\bar{C}_{i}-$ собственный прообраз кривой $C_{i}$ на $X$. Пусть $E_{1}, \ldots, E_{s}$ - все компоненты исключительного дивизора $\mathcal{D}$ разрешения. Обозначим через $\stackrel{\circ}{E}_{i}$ «неособую часть» компоненты $E_{i}$ в полном прообразе $\pi^{-1}(C)$ кривой $C$, т. е. $E_{i}$ без точек пересечения со всеми остальными компонентами полного прообраза $\pi^{-1}(C)$ кривой $C$. Пусть $\underline{m}_{i}=\left(m_{i 1}, \ldots, m_{i s}\right) \in \mathbb{Q}^{s} \geqslant 0$, $\underline{d}=\left(d_{1}, \ldots, d_{s}\right) \in \mathbb{Z}_{\geqslant 0}^{s}$ и одномерное представление $\alpha_{i}$ группы $G(i=1, \ldots, s)$ определены, как и выше. Те же аргументы, которые использовались при доказательстве теоремы 1, дают следующее утверждение.

Теорема 2. Эквивариантный ряд Пуанкаре $P^{G}\left(t_{1}, \ldots, t_{r}\right) r$-индексной фильтрации, определяемой набором нормирований $\left\{\widetilde{w}_{1}, \ldots, \widetilde{w}_{r}\right\}$, дается формулой

$$
P^{G}\left(t_{1}, \ldots, t_{r}\right)=\left.\left(\prod_{i=1}^{s}\left(1-\alpha_{i} \underline{T}^{\underline{d}} \underline{m}_{i}\right)^{-\chi\left(\stackrel{\circ}{E}_{i}\right)}\right)\right|_{T_{i} \mapsto \prod_{j:} \bar{C}_{j} \cap E_{i}=p t} t_{j}
$$

(здесъ $\underline{T}=\left(T_{1}, \ldots, T_{s}\right) ;$ в указанной подстановке $\prod_{j \in \varnothing} t_{j}$ предполагается равным единиие).

Авторы благодарны А. Г. Александрову за полезные замечания.

\section{ЛитЕРАТУРА}

[1] С. М. Гусейн-Заде, Ф. Дельгадо, А. Кампильо, Интегрирование по эйлеровой характеристике по пространству бункиий и полином Александера особенности плоской кривой, УМH, 55:6 (336) (2000), 127-128.

[2] A. Campillo, F. Delgado, S. M. Gusein-Zade, Poincaré series of a rational surface singularity, Invent. Math., 155 (2004), 41-53.

[3] A. Campillo, F. Delgado, S. M. Gusein-Zade, Poincaré series of curves on rational surface singularities, Comment. Math. Helv., 80:1 (2005), 95-102.

[4] A. Campillo, F. Delgado, S. M. Gusein-Zade, On Poincaré series of filtrations on equivariant functions of two variables, Moscow Math. J., 7:2 (2007), 243-255. 
[5] H. B. Laufer, Normal two-dimensional singularities, Ann. of Math. Stud., vol. 71, Princeton Univ. Press, Princeton, NJ; Univ. of Tokyo Press, Tokyo, 1971.

[6] W. D. Neumann, J. Wahl, Universal Abelian covers of surface singularities, in: Trends in singularities, Trends Math., Birkhauser, Basel, 2002, 181-190.

[7] T. Okuma, Universal Abelian covers of rational surface singularities., J. London Math. Soc. (2), 70:2 (2004), 307-324.

[8] T. Okuma, The geometric genus of splice-quotient singularities, http://arxiv.org/ abs/math/0610464.

[9] H. Pinkham, Singularités rationelles de surfaces, in: Séminaire sur les singularités des surfaces, Lecture Notes in Math., vol. 777, Springer-Verlag, Berlin-Heidelberg-New York, 1980, 147-178.

Московский государственный университет,

Поступило в редакцию механико-математический факультет

22 октября 2007 г.

e-mail: sabir@mccme.ru

University of Valladolid, Dept. of Algebra, Geometry and Topology

e-mail: campillo@agt.uva.es

University of Valladolid, Dept. of Algebra, Geometry and Topology

e-mail: fdelgado@agt.uva.es 